\title{
A Novel Mutant Mouse, joggle, with Inherited Ataxia
}

\author{
Ziyan CHEN $^{1,2)}$, Shizu HAYASAKA ${ }^{2)}$, Yoshiko TAKAGISHI ${ }^{2}$, \\ Yoshiharu Murata ${ }^{2}$, and Sen-ichi ODA ${ }^{1)}$
}

\begin{abstract}
1)Laboratory of Animal Management \& Resources, Graduate School of Bioagricultural Sciences, and ${ }^{2}$ Department of Teratology and Genetics, Research Institute of Environmental Medicine, Nagoya University, Furouchou, Chikusa-ku, Nagoya, Aichi 464-8601, Japan
\end{abstract}

\begin{abstract}
While establishing a new mouse strain, we discovered a novel mutant mouse that exhibited ataxia. Mating experiments showed that the mutant phenotype was due to a single autosomal recessive gene, which we have termed joggle (gene symbol: jog). The ataxia becomes apparent around postnatal day 12, when the mice first attempt to walk, and worsens thereafter. The life span of the mutant mouse is comparable to that of the wildtype mouse. After 21 days of age, the cerebellum weights of the jog/jog mice are significantly lower than those of the wild-type mice. These observations indicate that jog/jog mutant mice could be useful models for biomedical research.
\end{abstract}

Key words: ataxic, joggle, mutant mouse

The mouse is a valuable animal model for studies of mammalian genetics for several reasons which include fewer housing requirements owing to their small size and the availability of numerous coat colors and behavioral mutants [6]. Spontaneous or artificially induced mouse mutants with defects in function, morphology or development are important for biomedical studies. In particular, mutant strains of mice with ataxia, which exhibit cerebellum abnormalities, have been used for studies on human hereditary neurological diseases [3]. In the present report, we describe a novel ataxic mutant mouse (termed joggle) that inherits the genetic defect in a simple autosomal-recessive fashion.

The mouse breed colony used in this study, which was derived in 1985 from hybrids of inbred PW females and ENU-treated $\mathrm{C} 3 \mathrm{H} / \mathrm{He}$ males, was established at the Hatano Research Institute, Food and Drug Safety Center. This colony, which contains mice with dilute coat color and without joggle-like ataxic behavior, has been maintained at that institution as a source of noninbred strains for more than twenty years. Three mating pairs derived from the colony were supplied to us in 1998. During the breeding of these mice (tentative stock name of DSO), we discovered a joggle-like ataxic mouse strain. The ataxic mice were observed frequently among progeny produced by mating normal littermates of the partially inbred DSO strain. This observation suggested that ataxia is an inheritable trait, and we were able to create the novel mutant strain by brother-sister mating of heterozygous genotypes. The mutant was designated as joggle and the tentative gene symbol is jog, due to the observed phenotype of ataxic behavior

(Received 24 November 2005 / Accepted 28 March 2006)

Address corresponding: ZY. CHEN, Laboratory of Animal Management \& Resources, Graduate School of Bioagricultural Sciences, Nagoya University, Furouchou, Chikus-ku, Nagoya, Aichi 464-8601, Japan 
(Fig. 1). Subsequently, the members of the family of mutant mice were separated in an attempt to preserve the ataxic characteristic, and the forced heterozygous partially inbred DSO-jog/+ strain was established. However, this strain could not be maintained. Currently, the following three mouse strains are being maintained successfully (Fig. 2): (1) a partially inbred strain of DSO that lacks the joggle mutation and is undergoing the process of inbreeding; (2) a C57BL/6-

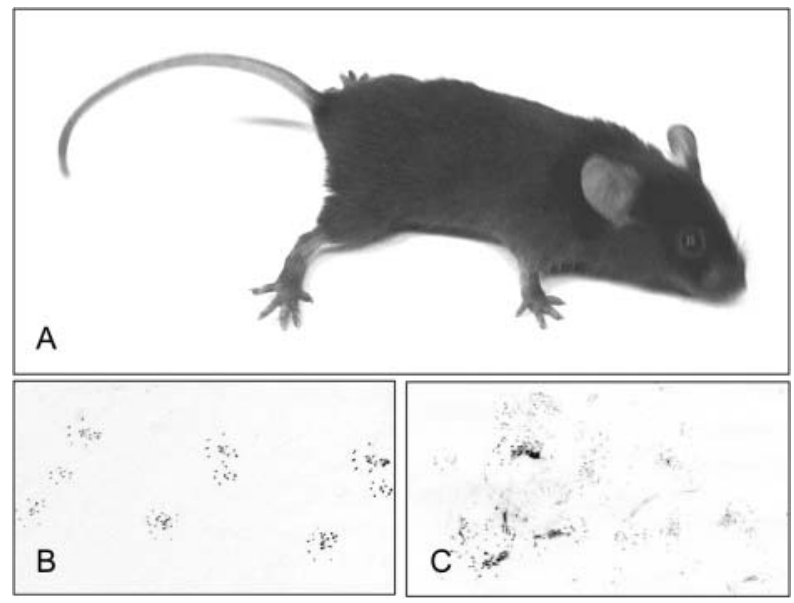

Fig. 1. The pathological manifestations of the joggle mouse. The motor deficit of the joggle mouse is characterized by gait instability, outward splaying of hind limbs, writhing of the trunk, and joggles when moving forward (A). C shows representative smudged footprints from a joggle mouse alongside those from a normal mouse (B).

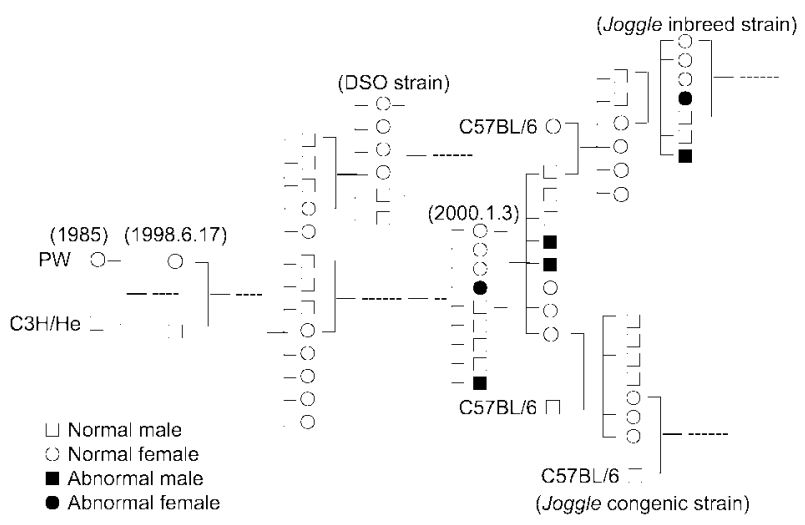

Fig. 2. Partial pedigree of the joggle mutant strain. During the breeding of the DSO strain mouse, we discovered the joggle-like ataxic mice. We are maintaining the joggle inbred strain and congenic strain by mating with C57BL/6. jog/+ partial congeneic strain that is in the process of backcross mating to $\mathrm{C} 57 \mathrm{BL} / 6$; (3) the partially inbred strain JOG, which is undergoing an inbreeding process using hybrids of DSO-jog/+ males and C57BL/6 females.

Since the mutant mice of both sexes are infertile, male and female heterozygous mice are bred to produce homozygous offspring, and pregnant females are checked daily for new litters. All of the mice are raised under conventional conditions and allowed access to a standard commercial diet and tap water ad libitum in our laboratory. Room temperature is maintained at 22 $\pm 1^{\circ} \mathrm{C}$, with a 12 -h light-dark cycle. Starting at postnatal day 12 (P12), homozygous mice can easily be distinguished from their heterozygous and wild-homozygous littermates based on the ataxic phenotype. The mice are weaned at P30 and maintained on a diet of dry rodent chow and water ad libitum. The experimental procedures were carried out in accordance with the Guidelines for Animal Experimentation of the Japanese Association for Laboratory Animal Science.

Ataxia first becomes apparent at about P12, at which time the mice attempt to walk, and gradually worsens thereafter. Motor disturbances are characterized by a hesitant joggling gait, difficulty in maintaining normal posture, inability to walk in a straight line, faulty coordination of the hind legs, writhing of the trunk, and step backward. The footprint patterns also show ataxia and gait abnormalities in the joggle mouse (Fig. 1). With development, an ataxic gait develops, with the hind limbs being more affected than the forelimbs. At three weeks of age, the symptoms become more severe and the mice display mild tremors of the trunk, with clasping of the hind legs when picked up by the tail. This condition persists throughout the life of the animal. Although the mutant mice are thinner than the normal mice, they do not have a reduced life span. The growth curves of the mutants and their littermates are shown in Fig. 3. The body-weights of the mutant mice are slightly lower than those of normal littermates at 12 days of age. From three weeks of age, the body-weights of the mutant mice differ significantly from those of normal mice. This divergence from the normal weight gain pattern is presumably due to difficulties experienced by the mutant mice in moving around and reaching either the nipples of the mother or the commercial diet and water. 


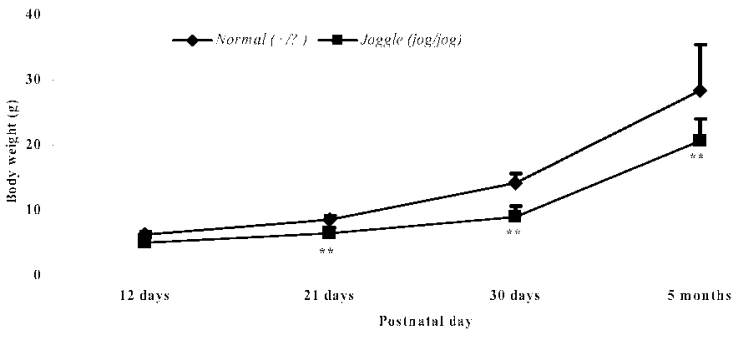

Fig. 3. Growth curves of joggle mice and their normal littermates $(\mathrm{n}=10)$. The body weights of the joggle mice are significantly lower than those of the wild-type mice from postnatal day 21 . Values represent the mean \pm SD. ${ }^{* *} P<0.01$, Student's $t$-test.

In general, the joggle mouse has a smaller body size than the wild-type mouse. The cerebellum of the joggle mouse is slightly smaller in size than the wild-type mouse, though the organization of the cerebellar cortex appears be normal (Fig. 4). The weights of the brain and cerebellum are similar for the two mice at 12 days of age. However, the ratio of cerebellar weight and brain weight shows that in the period from 3 weeks to 5 months of age, the mean weight of the brain, particularly that of the cerebellum, of the mutant mice is markedly lower than that of the normal mice (Fig. 5). A statistical analysis of variance demonstrated a significant main effect for group $(P<0.01)$. Under light microscopic examination, many dead cells were observed in the cerebellar cortex from P12 to 5 months in the joggle mice (data no show). Further analysis of morphological alteration of the cerebellum of the joggle mouse is in progress.

Our initial observations suggest that ataxia is a hereditary trait. The affected mice of both sexes are infertile, maybe due to their heavy ataxic behavior. Therefore, we maintained the phenotype by mating male and female heterozygous mice. The genetic analyses
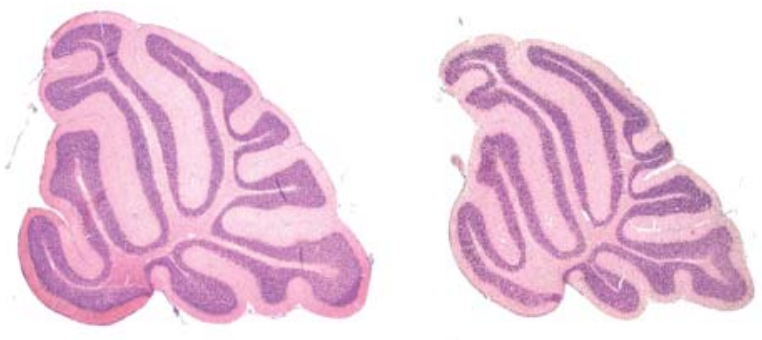

Fig. 4. Comparison of brains of wild-type and joggle mice from postnatal day 30. The brains of the joggle mice are smaller, most notably the cerebella of the mutant mice (right) are smaller than those of the wild-type mice. Scale bar $=100 \mu \mathrm{m}$.

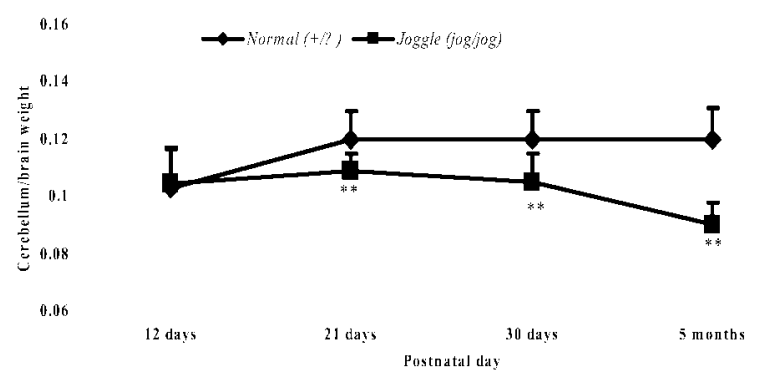

Fig. 5. The ratio of cerebellar weight and brain weight shows a severe decrease in cerebellar weight in joggle mice during growth $(\mathrm{n}=10) .{ }^{* *} P<0.01$, Student's $t$-test.

were conducted with $\mathrm{C} 57 \mathrm{BL} / 6$ as the normal control strain (Table 1). In test crosses with the mutant strain, mating of the C57BL/ $6 \times$ Carrier, Non-carrier $\times$ Carrier, Carrier $\times$ Non-carrier and Carrier $\times$ Carrier produced normal and mutant offspring in the ratios of 1:0 (57:0), 1:0 (193:0), 1:0 (127:0), and 3:1 (576:183), respectively. These results are in agreement with the expected ratios of Mendelian inheritance and show that the ataxia trait is controlled by a single autosomal re-

Table 1. Segregation of joggle mice in test crosses between Carrier mice and C57BL/6 mice

\begin{tabular}{|c|c|c|c|c|c|c|}
\hline \multicolumn{2}{|c|}{ Matings } & \multirow{2}{*}{$\begin{array}{c}\text { No. of } \\
\text { Progeny }\end{array}$} & \multicolumn{2}{|c|}{ No. of observed } & \multirow{2}{*}{$\begin{array}{l}\text { Expected seg- } \\
\text { regation ratio }\end{array}$} & \multirow{2}{*}{$\begin{array}{c}\mathrm{x}^{2} \\
\text { (Probability) }\end{array}$} \\
\hline Female $\quad \times$ & Male & & Normal & Abnormal & & \\
\hline C57BL/6 & Carrier & 57 & 57 & 0 & 1:0 & \\
\hline Non-carrier & Carrier & 193 & 193 & 0 & 1:0 & \\
\hline Carrier & Non-carrier & 127 & 127 & 0 & 1:0 & \\
\hline Carrier & Carrier & 759 & 576 & 183 & 3: 1 & $\begin{array}{c}0.320 \\
(0.5<P<0.6)\end{array}$ \\
\hline
\end{tabular}


cessive gene.

The behavioral phenotype of the joggle mouse is characterized by severe ataxia with gait instability and poor limb co-ordination; thus, it resembles the pogo mutant mouse and Wriggle Mouse Sagami [5, 7, 10]. The characteristic symptom of swaying suggested the joggle designation for this mutant mouse strain. In addition, when held by the tail, these mice display the "paw clasping" response [3,14], and this response is particularly pronounced in severely affected joggle mice. A complication of ataxia is that of tremors. For example, in weaver mutant mice, a high-frequency tremor is evident both at rest and during motion [8]. The joggle mutants also show mild tremors during movement. Interestingly, $p c d$ mutant mice and leaner mutant mice do not exhibit tremors $[12,13]$. Furthermore, the most important phenotypic differences between joggle and leaner mice [4] are that the joggle mice have earlier onset of clinical signs, similar to the Rolling mouse Nagoya strain $[9,11]$, and that the joggle mouse life span is normal.

Genetic studies of the newly discovered mutant show that the clinical traits are controlled by a single autosomal recessive gene. Therefore, we propose the designation of jog for the defective gene responsible for the joggle phenotype of these mice.

As ataxia is a major clinical feature, we suspect that the disorder might be neurological in origin, possibly a cerebellar abnormality. To date, approximately 100 mutations have been associated with neurological disorders in the mouse [2]. Studies on the different cerebellar mutants have contributed to a better understanding of the genetic and epigenetic factors that govern cerebellar development and function [1] by establishing the consequences of loss or defects in specific classes of cerebellar neurons. Likewise, further analyses of the defects in joggle mice may improve our understanding of human cerebellar diseases. It should be worthwhile to assess the usefulness of this novel ataxic mutant mouse as an animal model for biomedical studies. Studies on the mapping and identification of the jog gene are currently underway.

\section{Acknowledgments}

This work was supported by the Foundation of the 21st Century COE Program from the Ministry of Education, Culture, Sports, Science and Technology of Japan.

\section{References}

1. Duchala, C.S., Shick, H.E., Garcia, J., Deweese, D.M., Sun, X., Stewart, V.J., and Macklin, W.B. 2004. J. Comp. Neurol. 476: 113-129.

2. Green, M.C. 1989. pp.12-403. In: Genetic variants and strains of the laboratory mouse. (Lyon, M.F. ed.), Oxford University Press, Gustav Fischer Verlag, Stuttgart.

3. Grusser-Cornehls, U. and Baurle, J. 2001. Prog. Neurobiol. 63: 489-540.

4. Herrup, K.and Wilczynski, S.L. 1982. Neuroscience 7: 2185-2196.

5. Hyun, B.H., Kim, M.S., Choi, Y.K., Yoon, W.K., Suh, J.G., Jeong, Y.G., Park, S.K., and Lee, C.H. 2001. Mamm. Genome. 12: 250-252.

6. Jacob, H.J., Brown, D.M., Bunker, R.K., Daly, M.J., Dzau, V.J., Goodman, A., Koike, G., Kren, V., Kurtz, T., Lernmark, A., Levan, G., Mao, Y.P., Pettersson, A., Pravenec, M., Simon, J.S., Szpirer, C., Szporer, J., Trolliet, M.R., Winer, E.S., and Lander, E.S. 1995. Nature Genetics. 9: 63-69.

7. Jeong, Y.G., Hyun, B.H., and Hawkes, R. 2000. Brain Res. Dev. Brain Res. 125: 61-67.

8. Lane, P.W. 1965. pp.66-67. In: Catalog of the Neurological Mutations of the Mouse, (Sidman, R.L. ed.), Harvard University Press, Cambridge.

9. Oda, S. 1973. Exp. Anim. 22: 281-288.

10. Osawa, N. and Ohmura, M. 1992. Mouse Genome. 90: 223-225.

11. Sawada, K., Komatsu, S., Haga, H., Sun, X. Z., Hisano, S., and Fukui, Y. 1999. Brain Res. 829: 107-112.

12. Sidman, R.L., Green, M.C., and Appel, S.H. 1965. In: Catalog of the neurological mutants of the mouse, Harvard University Press, Cambridge.

13. Sidman, R.L., Lane, P.W., and Dickie, M.M. 1962. Science 137: 610-612.

14. Whishaw, I.Q. and Dunnett, S.B. 1985. Behav. Brain Res. 18: 11-29. 